\title{
Nearly Contraction Mapping Principle for Fixed Points of Hemicontinuous Mappings
}

\author{
Xavier Udo-utun, M. Y. Balla, and Z. U. Siddiqui \\ Department of Mathematics and Statistics, University of Maiduguri, Maiduguri, Nigeria \\ Correspondence should be addressed to M. Y. Balla; myballa@yahoo.com
}

Received 20 May 2013; Accepted 13 August 2013

Academic Editors: M. Hermann and K. Karamanos

Copyright ( 2013 Xavier Udo-utun et al. This is an open access article distributed under the Creative Commons Attribution License, which permits unrestricted use, distribution, and reproduction in any medium, provided the original work is properly cited.

We extend the application of nearly contraction mapping principle introduced by Sahu (2005) for existence of fixed points of demicontinuous mappings to certain hemicontinuous nearly Lipschitzian nonlinear mappings in Banach spaces. We have applied certain results due to Sahu (2005) to obtain conditions for existence-and to introduce an asymptotic iterative process for construction-of fixed points of these hemicontractions with respect to a new auxiliary operator.

\section{Introduction}

In this paper, we have applied certain results due to Sahu [1] on nearly contraction mapping principle to obtain conditions for existence of fixed points of certain hemicontinuous mappings and introduced an asymptotic iterative process for construction of fixed points of these hemicontinuous mappings with respect to a new auxiliary operator. Our results are important generalizations and an extension of important and fundamental aspect of a branch of asymptotic theory of fixed points of non-Lipschitzian nonlinear mappings in real Banach spaces.

Let $E$ and $Y$ be real Banach spaces and $K \subseteq E$ a nonempty subset of $E$. A mapping $T: K \rightarrow Y$ is said to be (see, e.g., [2])

(i) demicontinuous if whenever a sequence $\left\{x_{n}\right\} \subset X$ converges strongly to $x \in X$ it implies that the sequence $\left\{T x_{n}\right\}$ converges weakly to $T x \in Y$;

(ii) hemicontinuous if whenever a sequence $\left\{x_{n}\right\} \quad \mathrm{C}$ $X$ converges stronly on a line to $x \in X$ it implies that the sequence $\left\{T x_{n}\right\}$ converges weakly to $T x \in Y$, that is, $T\left(x_{0}+t_{n} x\right) \rightarrow T x_{0}$ as $t_{n} \rightarrow 0$.

Asymptotic fixed point theory which has been studied by so many authors $[1,3-6]$ has a fundamental role in nonlinear functional analysis concerning existence and construction of fixed points of Lipschitzian mappings, L-uniformly Lipschitzian mappings, and non-Lipschitzian mappings among other classes of operators (see, e.g., [5, 7-9]). A very important branch of the theory of asymptotic fixed point relates to the important class of asymptotically nonexpansive mappings which have been studied by various authors in specific types of Banach spaces.

Motivated by the need to relax continuity condition inherent in asymptotic nonexpansiveness of asymptotically nonexpansive mappings in certain applications, Sahu [1] considered and introduced the nearly contraction mapping principle into the study of asymptotic fixed point theory concerning nearly Lipschitzian mappings and obtained the following results among others.

Lemma 1. Let $C$ be a nonempty subset of a Banach space, and let $T: C \rightarrow C$ be hemicontinuous. Suppose that $T^{n} u=$ $p$ as $n \rightarrow \infty$ for some $u, p \in C$. Then, $p$ is an element of $\operatorname{Fix}(T)$, the set of fixed point of $T$.

Theorem 2. Let $C$ be a nonempty closed subset of a Banach space $X$ and $T: C \rightarrow C$ a demicontinuous nearly Lipschitzian mapping with sequence $\left\{\left(a_{n}, \eta\left(T^{n}\right)\right)\right\}$. Suppose $\eta_{\infty}(T)=$ $\overline{\lim }_{n \rightarrow \infty}\left[\eta\left(T^{n}\right)\right]^{1 / n}<1$. Then, we have the following:

(a) $T$ has unique fixed point $x^{*} \in C$; 
(b) for each $x_{0} \in C$, the sequence $\left\{T^{n} x_{0}\right\}$ converges strongly to $x^{*}$;

(c) $\left\|T^{n} x_{0}-x^{*}\right\| \leq\left(\left\|x_{0}-T x_{0}\right\| M\right) \sum_{i=n}^{\infty} \eta\left(T^{i}\right)$ for all $n \in \mathbb{N}$.

The aim of this work is appling Lemma 1 to obtain conditions for existence and uniqueness of asymptotic fixed point of a new auxiliary operator and appling Theorem 2 on the auxiliary operator to obtain an extension and a generalization of Theorem 2 which is a fundamental extension of important classical and related results.

\section{Preliminaries}

Let $K$ be a nonempty subset of a Banach space $E$ and $T$ : $K \rightarrow K$ a nonlinear mapping. The mapping $T$ is said to be Lipschitzian if for each $n \in \mathbb{N}$ there exists a constant $L_{n}>$ 0 such that $\left\|T^{n} x-T^{n} y\right\| \leq L_{n}\|x-y\|$ for all $x, y \in K$. A Lipschitzian mapping is called uniformly L-Lipschitzian if $L_{n}=L$ for all $n \in \mathbb{N}$ and asymptotically nonexpansive if $\lim _{n \rightarrow \infty} L_{n}=1$.

Next, let $K$ be a nonempty subset of a Banach space $E$ and $\left\{a_{n}\right\}$ a fixed sequence in $[0, \infty)$ with $a_{n} \rightarrow 0$ as $n \rightarrow$ $\infty$. A mapping $T: K \rightarrow K$ is called nearly Lipschitzian mapping with respect to $\left\{a_{n}\right\}$ if for each $n \in \mathbb{N}$ there exists a constant $L_{n} \geq 0$ such that

$$
\left\|T^{n} x-T^{n} y\right\| \leq L_{n}\left(\|x-y\|+a_{n}\right) .
$$

The infimum $\eta\left(T^{n}\right)=\sup \left\{\left\|T^{n} x-T^{n} y\right\| /\left(\|x-y\|+a_{n}\right) ; x, y \in\right.$ $K, x \neq y$ \} of constants $L_{n}$ for which (1) holds is called nearly Lipschitzian constant. Nearly Lipschitzian operators with sequences $\left\{\left(a_{n}, \eta\left(T^{n}\right)\right)\right\}$ are classified in $[1,2]$ as shown below:

(a) nearly contraction if $\eta\left(T^{n}\right)<1$ for all $n \in \mathbb{N}$;

(b) nearly nonexpansive if $\eta\left(T^{n}\right) \leq 1$ for all $n \in \mathbb{N}$;

(c) nearly asymptotically nonexpansive if $\eta\left(T^{n}\right) \geq 1$ for all $n \in \mathbb{N}$ and $\lim _{n \rightarrow \infty} \eta\left(T^{n}\right) \leq 1$;

(d) nearly uniformly L-Lipschitzian if $\eta\left(T^{n}\right) \leq L$ for all $n \in \mathbb{N}$;

(e) nearly uniformly L-contraction if $\eta\left(T^{n}\right) \leq L<1$ for all $n \in \mathbb{N}$.

Examples and a short survey of these classes of nearly Lipschitzian operators are listed above, and related operators are illustrated in [1] (pp. 655-656) where it is remarked that if $K$ is bounded then the asymptotically nonexpansive mapping $T$ is a nearly nonexpansive mapping. Also, it is observed therein that a nearly asymptotically nonexpansive mapping reduces to asymptotically nonexpansive type if $K$ is bounded. For details authors are referred to Agarwal et al. [2] pp. 259-263, especially the bibliographic notes and remarks there in.

\section{Main Results}

Our main results depend on Lemma 1 and the following new important inequality, needed in the sequel, which we shall prove using archimedean property. We are still sharpening an estimate for the parameter $\tau$ in Lemma 3 below.
Lemma 3. Let $V$ be a normed linear space over, a scaler field $\mathbb{F}(\mathbb{F}$ is real or complex). Then, for all distinct points $x, y \in$ $V$ there exists $\tau \in \mathbb{R}$ such that

$$
\|\alpha x-\beta y\| \leq[2|\alpha|+\tau|\beta|]\|x-y\|
$$

for all $\alpha, \beta \in \mathbb{F}$.

Proof. As mentioned above, the proof is a consequence of Archimedean property of real numbers that if $a$ and $b$ are positive real numbers then $a<n b$ for some $n \in \mathbb{N}$. Since $x \neq y$, we have

$$
\begin{aligned}
(\alpha+\beta)(x-y) & =\alpha x-\beta y+\beta x-\alpha x \\
& =\alpha x-\beta y-(\alpha y-\beta x) \\
\Longrightarrow|\alpha+\beta|\|x-y\| & =\|\alpha x-\beta y-(\alpha y-\beta x)\| \\
& \geq\|\alpha x-\beta y\|-\|\alpha y-\beta x\| \\
\Longrightarrow\|\alpha x-\beta y\| \leq & |\alpha+\beta|\|x-y\|+\|\beta x-\alpha y\| \\
& =|\alpha+\beta|\|x-y\|+\left\|\left(\alpha+\beta_{1}\right) x-\alpha y\right\| \\
& \leq|\alpha+\beta|\|x-y\|+|\alpha|\|x-y\|+\left|\beta_{1}\right|\|x\| \\
\leq & (2|\alpha|+\tau|\beta|)\|x-y\| .
\end{aligned}
$$

Equation (3) follows from Archimedean property while boundedness is inferred from the fact that $\beta_{1}=\beta-\alpha$ for arbitrary $\alpha, \beta \in \mathbb{F}$.

Remark 4. It is important to make the following observations.

(1) If $\alpha, \beta \in \mathbb{R}$ then (3) reduces to

$$
\|\alpha x-\beta y\| \leq 2(|\alpha|+|\beta|)\|x-y\|,
$$

as verified below: since $\alpha, \beta \in \mathbb{R}$, if on the contrary (4) is not satisfied then from (3) we have $\left|\beta_{1}\right|\|x\|>$ $|\beta|\|x-y\|$ which end up with a contradiction demonstrated below.

Suppose $\left|\beta_{1}\right|\|x\|>|\beta|\|x-y\|$. Setting $\alpha, \beta>0$ such that $\beta-\alpha>0$ yields $\alpha>1$ whenever $\alpha, \beta>0$ such that $\beta-\alpha>0$ which is a contradiction.

(2) It is important to observe that if $x$ and $y$ were not distinct in Lemma 3 then $\alpha=\beta$ would be a valid and natural constraint. However, for $x=y$ the problem is trivial.

Lemma 5. Let $K$ be a nonempty subset of a Banach space, and let $T: K \rightarrow K$ be a nearly Lipschitzian map with sequence $\left\{\left(a_{n}, \eta\left(T^{n}\right)\right)\right\}$ such that $\eta_{\infty}(T)=\varlimsup_{n \rightarrow \infty}\left[\eta\left(T^{n}\right)\right]^{1 / n}<1$. Then the auxiliary operator $S: \mathbb{N} \times K \rightarrow K$ defined by $S(n, x)=$ $T^{n-1} x+\left\|T^{n} x-T^{n-1} x\right\| x$ has a fixed point in $E$. 
Proof. Given that $S(n, x)=T^{n-1} x+\left\|T^{n} x-T^{n-1} x\right\| x$ where $T$ is a nearly Lipschitzian map with sequence $\left\{\left(a_{n}, \eta\left(T^{n}\right)\right)\right\}$, we have

$$
\begin{aligned}
&\|S(n, x)-S(n+1, x)\| \\
& \leq\left\|T^{n} x-T^{n-1} x\right\| \\
&+\|\| T^{n} x-T^{n-1} x\|x-\| T^{n+1} x-T^{n} x\|x\| \\
& \leq \eta\left(T^{n-1}\right)\left(\|T x-x\|+a_{n-1}\right) \\
&+\|\| T\left(T^{n} x\right)-T\left(T^{n-1} x\right)\|x-\| T^{n} x-T^{n-1} x\|x\| \\
& \leq \eta\left(T^{n-1}\right)\left(\|T x-x\|+a_{n-1}\right) \\
&+\left\|\eta(T)\left(\left\|T^{n} x-T^{n-1} x\right\|+a_{1}\right) x-\right\| T^{n} x-T^{n-1} x\|x\| \\
& \leq \eta\left(T^{n-1}\right)\left(\|T x-x\|+a_{n-1}\right) \\
&+\left\|\left[\eta(T) a_{1}+(\eta(T)-1)\left\|T^{n} x-T^{n-1} x\right\|\right] x\right\| \\
& \leq \eta\left(T^{n-1}\right)\left(\|T x-x\|+a_{n-1}\right) \\
&+\left[\eta(T) a_{1}+\eta\left(T^{n-1}\right)(\eta(T)-1)\left(\|T x-x\|+a_{n-1}\right)\right] \\
& \times\|x\| .
\end{aligned}
$$

This gives $\left\|S^{n} x-S^{n+1} x\right\| \leq \eta(T) a_{1}\|x\|+[1+(\eta(T)-$ 1) $\|x\|] \eta\left(T^{n-1}\right)\left(\|T x-x\|+a_{n-1}\right)$ which yields $\left\|S^{n} x-S^{n+1} x\right\| \leq$ $\eta(T) a_{1}\|x\|+[1+(\eta(T)-1)\|x\|]\left(d_{0_{x}}+M\right) \eta\left(T^{n-1}\right)$ where

$$
d_{n_{x}}=\left\|T^{n+1} x-T^{n} x\right\| .
$$

Using the hypothesis

$$
\eta_{\infty}(T)=\varlimsup_{n \rightarrow \infty}\left[\eta\left(T^{n}\right)\right]^{1 / n}<1
$$

together with the Root Test for convergence of series of real numbers, we obtain $\sum_{n=1}^{\infty}\left\|S^{n} x-S^{n+1} x\right\|<\infty$ which means the sequence $\{S(n, x)\}$ is a Cauchy sequence and so has a limit point $x^{*}$ in $E$.

We are left to show that the limit $x^{*}$ of $\left\{x_{n}\right\}=\{S(n, x)\}$ is a fixed point of $S(n, \cdot)$, for all $n \in \mathbb{N}$. To achieve this, it suffices to prove that $S(n, \cdot)$ is continuous which follows an application of Lemma 3, namely. Let $x, y \in K$, then

$$
\begin{aligned}
& \|S(\cdot, x)-S(\cdot, y)\| \\
& =\|x+\| T x-x\|x-y-\| T y-y\|y\| \\
& \leq\|x-y\|+\|\| T x-x\|x-\| T y-y\|y\| \\
& =\|x-y\|+\left\|d_{1_{x}} x-d_{1_{y}} y\right\| \\
& \leq\left[d_{1_{x}}+\tau d_{1_{y}}+1\right]\|x-y\|
\end{aligned}
$$

(by Lemma 3 and (8)) for some positive real number $\tau$. So given any $\epsilon$, we have $\delta=$ $\epsilon /\left(d_{1_{x}}+\tau d_{1_{y}}+1\right)$ such that $\|S x-S y\|<\epsilon$ whenever $\| x-$ $y \|<\delta$ for some $\tau>0$. Therefore, $S$ is continuous in $x$ and so $\lim _{n \rightarrow \infty} x_{n}=\lim _{n \rightarrow \infty} S(n, x)=S\left(n, \lim _{n \rightarrow \infty} x_{n}\right)=x^{*}$.

To apply Lemma 1, we need its extension for hemicontinuous mappings given in the following form.

Lemma 6. Let $K$ be a nonempty subset of a Banach space, and let $T: K \rightarrow K$ be hemicontinuous nearly Lipschitzian mapping. Suppose that $T^{n} u=p$ as $n \rightarrow \infty$ for some $u, p \in K$. Then, $p$ is an element of $\operatorname{Fix}(T)$.

Proof. Consider the following operator $\mathcal{S}: \mathbb{N} \times E \rightarrow E$ defined by

$$
\mathcal{S}(n, u)=u+\left\|T^{n} u-T^{n-1} u\right\| T^{n-1} u
$$

Clearly, $\mathcal{S}$ restricted to $K$ reduces to the auxiliary operator $S$ above at the fixed point of $\mathcal{S}$. We will show that given that $T$ is hemicontinuous then $\mathcal{S}$ is a selfmap of $K$ for all $n$, that is, $\mathcal{S}(\cdot, x): K \rightarrow K$ since $K$ is closed.

Clearly, $\delta$ restricted to $K$ and $T$ have common fixed point set, that is, $\operatorname{Fix}(T)=\operatorname{Fix}(S)$ (provided $T$ has a fixed point) and $S(n+1, u)=u+\left\|T^{n+1} u-T^{n} u\right\| T^{n} u=u+d_{n} T^{n} u$. But from the last proof, we verified that $S$ is a continuous mapping on $K$ and has asymptotic fixed point $x^{*} \in E$. Also, by hemicontinuity of $T$ and continuity of $\mathcal{S}$ the sequence $f x_{n} g=f \mathcal{S}\left(n ; x_{0} g\right)$ converges strongly to $x$ which means that $f \mathcal{S}\left(n ; x_{n}\right) g$ converges weakly to $\mathcal{S}(n ; p)$ which means $\mathcal{S}$ is demicontinuous on $K$.

By Lemma 1, we have that $p \in \operatorname{Fix}(\mathcal{S})=\operatorname{Fix}(T)$.

Theorem 7. Let $K$ be a nonempty closed subset of a Banach space $E$ and $T: K \rightarrow K$ a hemicontinuous nearly Lipschitzian mapping with sequence $\left\{\left(a_{n}, \eta\left(T^{n}\right)\right)\right\}$. Suppose $\eta_{\infty}(T)=$ $\varlimsup_{n \rightarrow \infty}\left[\eta\left(T^{n}\right)\right]^{1 / n}<1$. Then, we have the following:

(a) $T$ has unique fixed point $p \in K$;

(b) for each $x_{0} \in K$, the sequence $\left\{T^{n} x_{0}\right\}$ converges strongly to $p$;

(c) $\left\|S\left(n, x_{0}\right)-p\right\| \leq\left[2 M+(\eta(T)+1)\left\|x_{0}-T x_{0}\right\|\right] \sum_{i=n}^{\infty} \eta\left(T^{i}\right)$ for all $n \in \mathbb{N}$ where $M=\sup _{n \in \mathbb{N}} a_{n}$ and $S(n, x)=$ $T^{n-1} x+\left\|T^{n} x-T^{n-1} x\right\| x$.

Proof. By Lemma 6, the auxiliary operator given by $\mathrm{Su}=$ $u+\|T u-u\| u$ is a selfmap of $K$, and together with Lemma 5 we conclude that $S$ has a fixed point in $K$ which is also a fixed point of $T$. To prove (a), we are left to show that the fixed point is unique. The proof of uniqueness and for (b) and (c) follow from the fact that $\mathcal{S}$ is demicontinuous contraction so that Theorem 2 applies.

\section{References}

[1] D. R. Sahu, "Fixed points of demicontinuous nearly Lipschitzian mappings in Banach spaces," Commentationes Mathematicae Universitatis Carolinae, vol. 46, no. 4, pp. 653-666, 2005. 
[2] R. P. Agarwal, D. O'Regan, and D. R. Sahu, Fixed Point Theory for Lipschitzian-Type Mappings with Applications, Springer Science+Business, New York, NY, USA, 2009.

[3] R. E. Bruck, "On the convex approximation property and the asymptotic behavior of nonlinear contractions in Banach spaces," Israel Journal of Mathematics, vol. 38, no. 4, pp. 304314, 1981.

[4] K. Goebel and W. A. Kirk, "A fixed point theorem for transformations whose iterates have uniform Lipschitz constant," Studia Mathematica, vol. 47, pp. 135-140, 1973.

[5] K. Goebel and W. A. Kirk, Topics in Metric Fixed Point Theory, Cambridge University Press, Cambidge, UK, 1990.

[6] D. R. Sahu and J. S. Jung, "Fixed-point iteration processes for non-lipschitzian mappings of asymptotically quasinonexpansive type," International Journal of Mathematics and Mathematical Sciences, vol. 2003, no. 33, pp. 2075-2081, 2003.

[7] T. D. Benavides, G. L. Acedo, and H. K. Xu, "Weak uniform normal structure and iterative fixed points of nonexpansive mappings," Colloquium Mathematicum, vol. 68, no. 1, pp. 17-23, 1995.

[8] F. E. Browder, "Fixed point theorems for noncompact mappings in Hilbert spaces," Proceedings of the National Academy of Sciences of the United States of America, vol. 53, no. 6, pp. 12721276, 1965.

[9] K. Goebel and W. A. Kirk, "A fixed point theorem for asymptotically nonex- pansive mappings," Proceedings of the American Mathematical Society, vol. 35, no. 1, pp. 171-174, 1972. 


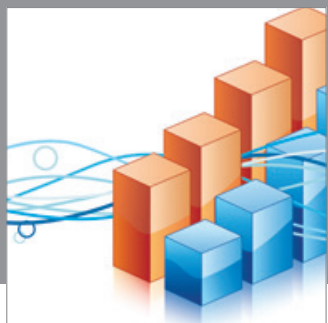

Advances in

Operations Research

mansans

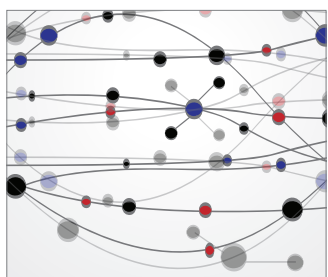

The Scientific World Journal
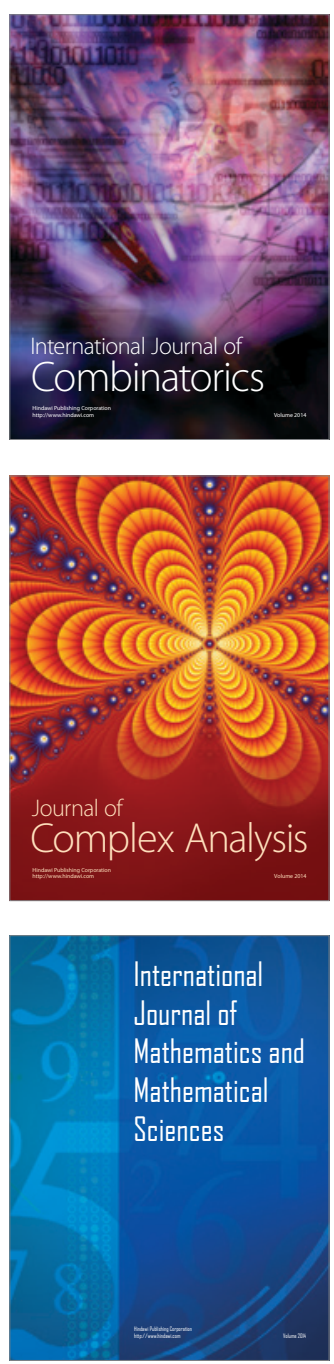
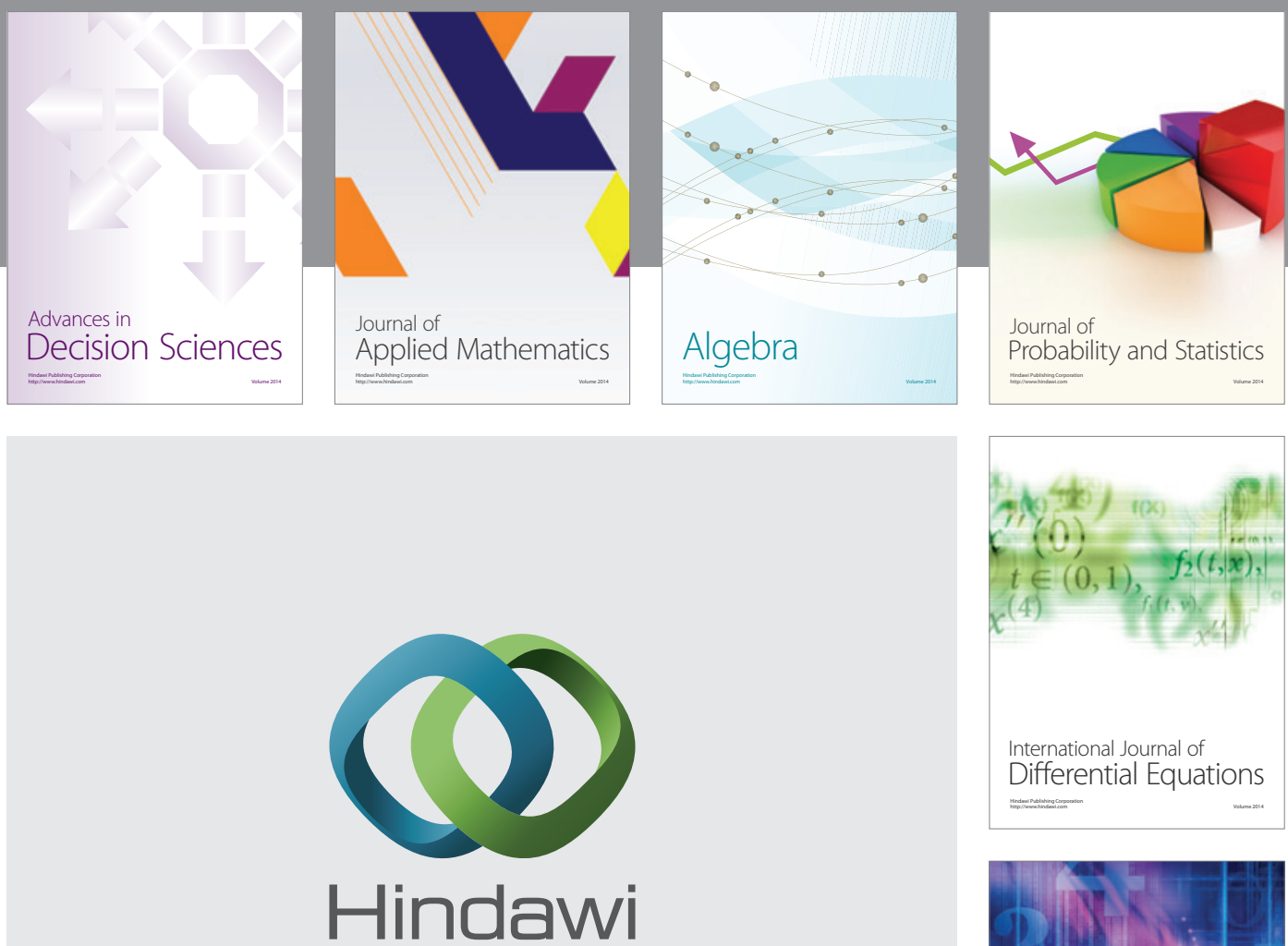

Submit your manuscripts at http://www.hindawi.com
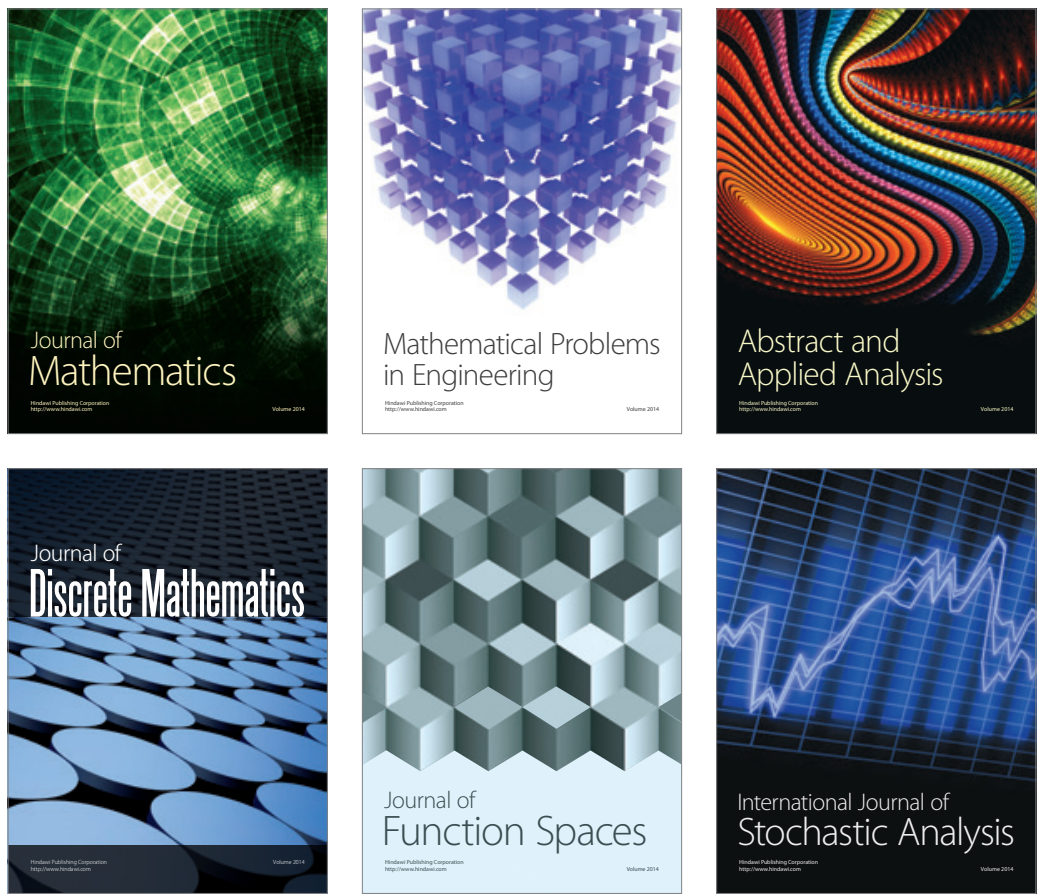

Journal of

Function Spaces

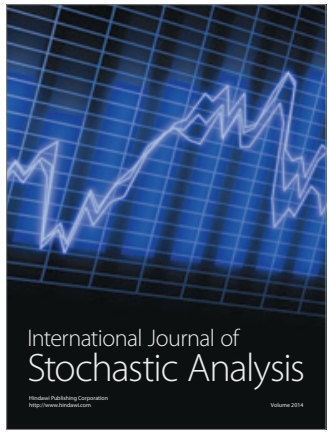

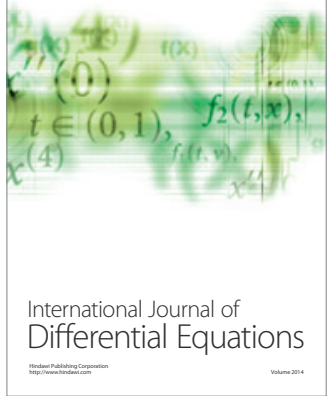
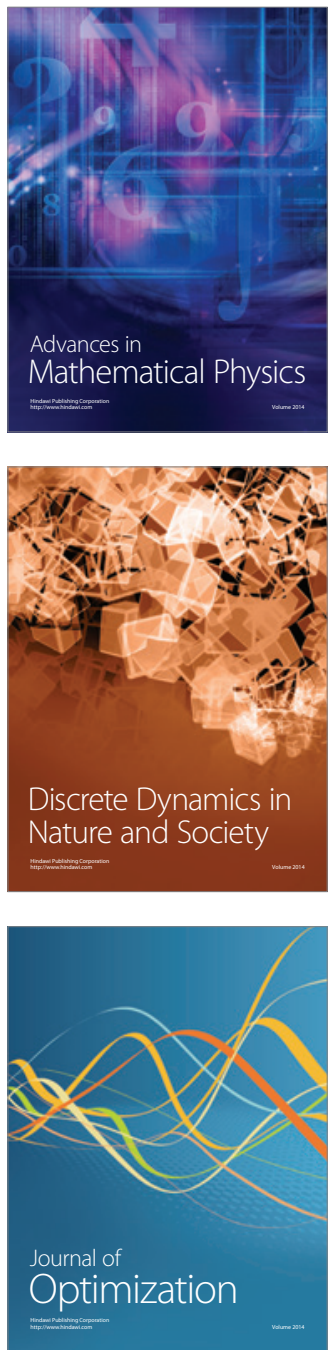